\title{
Un estudio observacional no participante sobre el uso de máquinas tragaperras en el norte de España
}

\section{A non-participant naturalistic observational study on the use of slot machines in northern Spain}

\author{
Aris Grande-Gosende*, Víctor Martínez-Loredo*, Eduardo García-Cueto*, \\ José Ramón Fernández-Hermida*. \\ * Facultad de Psicología. Universidad de Oviedo.
}

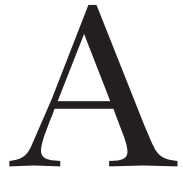
proximadamente un $1,5 \%$ de la población adulta presenta trastorno de juego patológico (JP) (Gowing et al., 2015), siendo las máquinas tragaperras una de las actividades de juego más adictivas (Calado y Griffiths, 2016). La reglamentación española permite a los restaurantes y bares operar máquinas de azar tipo B, existiendo más de 180,000 máquinas en España (Dirección General de Ordenación del Juego, 2016). Casi todos los locales de restauración tienen al menos una. A pesar de la preocupación por su alta accesibilidad hacia colectivos más vulnerables (menores, jugadores problemáticos), se desconoce el número de usuarios de máquinas de azar y sus características asociadas. Este estudio tiene como fin presentar, por primera vez, una estimación del uso de máquinas de azar a nivel poblacional y sus características asociadas.

Para la realización del estudio observacional no participativo, se llevó a cabo un muestreo aleatorizado por conglomerados bietápicos, usando los registros oficiales de locales de hostelería con licencia para operar máquinas de azar en Asturias. Los conglomerados se crearon teniendo en cuenta: zona geográfica (zonas costeras vs. de interior), ámbito (rural vs. urbano), sector económico (primario, secundario o terciario) y número de máquinas de azar por local (una vs. dos). Las zonas seleccionadas representaban el $66,05 \%$ de la totalidad de las máquinas de azar en Asturias: Oviedo, Gijón, Avilés, Ribadesella, Pravia, Pola de Siero y las cuencas mineras (Mieres, Langreo, La Felguera, Sama, Ciaño, Lada y Riaño). Se dividió cada zona en locales con una o dos máquinas. Según el número total de locales que operaban máquinas de azar electrónicas (MAE) en cada conglomerado, se visitaron 55 locales de hostelería durante tres periodos temporales (mañana: 8-12h; tarde: 12-16h; noche: 16-22h). Siguiendo un estudio previo (Dirección de Juego y Espectáculos del Departamento de Interior del Gobierno Vasco, 2009), se realizó una sesión observacional de 60 minutos en cada local (165 sesiones en total) por parte de dos experimentadores nivel Máster especialmente entrenados para ello. No fue necesaria la aprobación del comité de ética dada la naturaleza observacional del estudio sobre la conducta manifiesta (Organización Mundial de la Salud, 2016).

Se clasificó a los participantes por sexo y tres grupos de edad: < 18, 18-25, 26-35, 36-50, + 50 años de edad. Se estimó el consumo de bebidas (ninguna, bebida sin alcohol, bebida con alcohol o ambas) y el nivel de intoxicación alcohólica aparente. Se valoraron cinco índices de comportamiento de juego: Persistencia (juego persistente/intermitente), tiempo dedicado al juego sin pausa por ocasión, acompañamiento (solo/acompañado), cambio entre máquinas de azar en locales con dos máquinas, urgencia al acceder al local (acceso directo/tras pedir una bebida).

Se realizaron pruebas Chi-cuadrado con corrección de Bonferroni para analizar diferencias demográficas y de las conductas de juego. Para estimar la prevalencia del uso de máquinas de azar, se utilizaron los datos censales de cada una de las siete áreas seleccionadas (Instituto Nacional de Estadística, 2016), comprendiendo 622.663 (59,72\%) del total de 1.042.608 habitantes en la región. Del total de 3.502 máquinas de azar en los espacios públicos, se inclu-

Recibido: Marzo 2019; Aceptado: Julio 2019.

Enviar correspondencia a: Victor Martinez-Loredo

Unidad Clínica de Conductas Adictivas, Facultad de Psicología. Universidad de Oviedo. Pza Feijoo s/n. 33003 Oviedo-España. Tel. +34 985 104189 E-mail: martinezlvictor@uniovi.es loredo@cop.es 
yeron $74(2,11 \%)$ en este estudio (38 máquinas de azar en locales con dos máquinas). Dada la naturaleza binaria de la variable observada con distribución binomial y varianza máxima de 0,25, el error máximo fue $\pm 1,08(\alpha=.05)$.

Se registraron a 89 usuarios durante el protocolo de observación (Tabla 1). Dada la prevalencia baja de usuarios menores de 25 años, se fusionaron los primeros dos grupos en una misma categoría: 18-35 años de edad $(\mathrm{n}=17$, $19,1 \%)$. Los resultados principales mostraron que mientras que era más probable que el grupo más joven jugara acompañado, el grupo de más edad acostumbraba jugar a solas $\left(\chi^{2}(2)=10,34, p=, 006, V\right.$ de Cramer = ,34). También, era más probable que los jugadores solitarios apostasen con una mayor persistencia $\left(\chi^{2}(1)=7,3, \mathrm{p}=, 007, \Phi=, 33\right)$.

Tabla 1. Características de la muestra de usuarios de máquinas de azar

\begin{tabular}{|c|c|}
\hline Variables & n (\%) \\
\hline Sexo (hombre) & $84(94,4)$ \\
\hline \multicolumn{2}{|l|}{ Edad } \\
\hline 18-25 años & $2(2,2)$ \\
\hline $26-35$ años & $15(16,9)$ \\
\hline 50 o más años & $26(29,2)$ \\
\hline \multicolumn{2}{|l|}{ Zona geográfica } \\
\hline Oviedo & $13(14,6)$ \\
\hline Gijón & $20(22,5)$ \\
\hline Avilés & $14(15,7)$ \\
\hline Ribadesella & $2(202)$ \\
\hline Pravia & $8(9)$ \\
\hline Pola de Siero & $14(15,7)$ \\
\hline Cuencas mineras & $18(20,2)$ \\
\hline Día de juego (día hábil) & $46(51,7)$ \\
\hline \multicolumn{2}{|l|}{ Periodo de juego } \\
\hline Mañana & $33(37,1)$ \\
\hline Tarde & $33(37,1)$ \\
\hline Noche & $23(25,8)$ \\
\hline Tiempo dedicado al juego (minutos) ${ }^{a}$ & $89(5)$ \\
\hline Persistencia en el juego (persistente) & $73(82)$ \\
\hline Acompañamiento (solo) & $77(86,5)$ \\
\hline Cambio entre máquinas (no) ${ }^{b}$ & $46(85,2)$ \\
\hline Uso de MAE (tras pedir una bebida) ${ }^{c}$ & $64(71,9)$ \\
\hline \multicolumn{2}{|l|}{ Tipo de bebidad } \\
\hline Ninguna & $8(9,2)$ \\
\hline Sin alcohol & $33(37,9)$ \\
\hline Con alcohol & $46(52,8)$ \\
\hline Intoxicación alcohólica (no) & $85(95,5)$ \\
\hline
\end{tabular}

Nota. ${ }^{\text {a Media. }}{ }^{\mathrm{b}}$ Considerando solo los conglomerados con dos máquinas de azar. ${ }^{\mathrm{C}} 11$ participantes omitidos debido a dificultades en el proceso observacional. ${ }^{\circledR} 2$ participantes omitidos debido a dificultades en el proceso observacional. $M A E=$ Máquina de azar electrónica.

En base a los usuarios observados y los habitantes registrados en cada zona, la prevalencia estimada del uso de máquinas de azar en Asturias fue de aproximadamente 44.637 jugadores (4,28\% de la población total). Aunque la mayoría de los jugadores estimados residían en las dos ciudades más grandes (Gijón: $\mathrm{n}=7.285$; Oviedo: $\mathrm{n}=4.940$ ), Pravia $(9,88 \%)$ y las cuencas mineras $(8,56 \%)$ tuvieron las proporciones más altas de jugadores de máquinas de azar per cápita. Oviedo tuvo la proporción más baja (2,62\%). La mayoría de los usuarios eran hombres ( $\mathrm{n}=42.129$, $94,38 \%)$ con edades de entre 26-35 años $(n=4.969,6,13 \%$ de los residentes de esta franja de edad). Teniendo en cuenta sexo y edad, había más hombres en el grupo de 2635 años $(\mathrm{n}=4.969,12,35 \%$ del total de residentes hombres en dicha franja de edad) y más mujeres en el grupo de 3650 años $(n=994,1,12 \%$ del total de residentes mujeres en dicha franja de edad).

De este estudio se derivan varias implicaciones a nivel preventivo. Un sinfín de estudios ha apuntado hacia la reducción del potencial adictivo de las MAE mediante la modificación de algunas características estructurales (Griffiths y Auer, 2012). La instalación de identificadores electrónicos personales para activar las máquinas de azar reduciría la accesibilidad a las MAE y prevendría sesiones prolongadas de juego (Rockloff, Donaldson, y Browne, 2015). A la vez, se podría registrar datos relacionados con el juego y lanzar mensajes de retroalimentación (Monaghan, 2008). Otras estrategias preventivas ambientales recomendadas incluyen reducir el número de locales con licencia para operar las MAE según su ubicación cercana a colegios o centros de salud, o incluso someter las MAE a un monopolio estatal de juego (Rossow y Hansen, 2016).

\section{Reconocimientos}

Este estudio formó parte de un informe encargado por el Consejo del Juego del Principado de Asturias, y recibió apoyo de parte del Consejo de Salud del Principado de Asturias (FUO-205-17). La entidad financiadora no asumió papel alguno en el diseño, la recolección de datos o el análisis de los resultados de este estudio.

\section{Conflicto de interés}

Los autores declaran la inexistencia de conflicto de interés.

\section{Referencias}

Calado, F. y Griffiths, M. D. (2016). Problem gambling worldwide: An update and systematic review of empirical research (2000-2015). Journal of Behavioral Addiction, 5, 592-613. doi: 10.1556/2006.5.2016.073.

Dirección de Juego y Espectáculos del Departamento de Interior del Gobierno Vasco. (2009). Estudio Sociológico sobre el Juego y sus Patologías y Conductas Adictivas en la Comunidad Autónoma de Euskadi [Sociological study on gambling, its pathologies, and addictive behaviors in the Autonomous Community of Euskadi]. Recuperado de http://www.euskadi.eus/informacion/publicaciones-de-juego-y-espectaculos/web01-a2joko/es/. 
Dirección General de Ordenación del Juego. (2016). Memoria Anual 2016 [Annual Report 2016]. Madrid: Ministerio de Hacienda y Administraciones Públicas.

Gowing, L. R., Ali, R. L., Allsop, S., Marsden, J., Turf, E. E., West, R. y Witton, J. (2015). Global statistics on addictive behaviours: 2014 status report. Addiction, 110, 904-919. doi: $10.1111 /$ add.12899.

Griffiths, M. D. y Auer, M. (2012). The irrelevancy of game-type in the acquisition, development, and maintenance of problem gambling. Frontiers in Psychology, 3, 621. doi: 10.3389/fpsyg.2012.00621.

Instituto Nacional de Estadística. (2016). Official Population Figures referring to revision of Municipal Register. Recuperado de www.ine.es/jaxiT3/Tabla.htm?t=2886\&L=0.
Monaghan, S. (2008). Review of pop-up messages on electronic gaming machines as a proposed responsible gambling strategy. International Journal of Mental Health and Addiction, 6, 214-222. doi: 10.1007/s11469-007-9133-1.

Organización Mundial de la Salud. (2016). International Ethical Guidelines for Health-related Research Involving $\mathrm{Hu}$ mans. Recuperado de https://cioms.ch/wp-content/ uploads/2017/01/WEB-CIOMS-EthicalGuidelines.pdf.

Rockloff, M. J., Donaldson, P. y Browne, M. (2015). Jackpot Expiry: An Experimental Investigation of a New EGM Player-Protection Feature. Journal of Gambling Studies, 31, 1505-1514. doi: 10.1007/s10899-014-9472-3.

Rossow, I. y Bang Hansen, M. (2016). Gambling and gambling policy in Norway-an exceptional case. Addiction, 111, 593-598. doi: 10.1111/add.13172. 
Un estudio observacional no participante sobre el uso de máquinas tragaperras en el norte de España 